\title{
Control of Aptamer Function Using Radiofrequency Magnetic Field
}

\author{
Kenichi Taira, ${ }^{1}$ Koichi Abe, ${ }^{1}$ Takayuki Ishibasi, ${ }^{2,3}$ Katsuaki Sato, ${ }^{2,4}$ and Kazunori Ikebukuro ${ }^{1}$ \\ ${ }^{1}$ Department of Biotechnology, Graduate School of Engineering, Tokyo University of Agriculture and Technology, \\ Tokyo 184-8588, Japan \\ ${ }^{2}$ Department of Applied Physics, Graduate School of Engineering, Tokyo University of Agriculture and Technology, \\ Tokyo 184-8588, Japan \\ ${ }^{3}$ Department of Material Science and Technology, Nagaoka University of Technology 1603-1, Kamitomioka, \\ Nagaoka, Niigata 940-2188, Japan \\ ${ }^{4}$ PRESTO Program, Japan Science and Technology Agency, 5 Sambancho, Chiyodaku, Tokyo 102-0075, Japan \\ Correspondence should be addressed to Kazunori Ikebukuro, ikebu@cc.tuat.ac.jp \\ Received 22 May 2011; Accepted 28 June 2011 \\ Academic Editor: Daisuke Miyoshi \\ Copyright () 2011 Kenichi Taira et al. This is an open access article distributed under the Creative Commons Attribution License, \\ which permits unrestricted use, distribution, and reproduction in any medium, provided the original work is properly cited.
}

Remote control of aptamer function has allowed us to control protein function in space and time. Here, we propose a novel control system for aptamer function by radiofrequency magnetic field- (RFMF-) induced local heating of a gold nanoparticle conjugated with an aptamer. In this study, we used a 31-mer thrombin-binding aptamer (TBA), which can inhibit thrombin activity, as a model aptamer. We evaluated the RFMF control of the inhibitory activity of a gold nanoparticle-conjugated TBA. To evaluate the effect of RFMF on enzymatic activity, we utilized a complementary DNA strand that maintains the broken structure during the activity assay. We observed a decrease in the inhibitory activity of TBA after RFMF irradiation. It indicates that RFMF is capable of controlling the TBA structure. Because RFMF allows noninvasive control of aptamer function, this strategy is expected to be novel way of controlling aptamer drug activity.

\section{Introduction}

Aptamers, single-stranded DNA or RNA molecules, recognize various target molecules on forming a particular threedimensional structure $[1,2]$. Each aptamer has a different topology and three-dimensional structure that recognizes a target [3]. Although most proteins lose their function by denaturing their structure irreversibly, aptamers can form correct structures reversibly even after denaturation of their structure. Therefore, aptamer function can be controlled reversibly by controlling the structures.

Many researchers have reported the remote control of functional nucleic acids by UV light irradiation. Caged compounds, which can be controlled by light, have been utilized for the remote control of aptamers and siRNA $[4,5]$. However, because only a small amount of UV light penetrates deep tissues and also damages DNA structure, the application of UV light would be limited unless specific devices are used that generate two-photon absorption [6].

Hamad-Schfferil et al. have reported remote electronic control of the DNA structure by inductive coupling of a radiofrequency magnetic field to a metal nanocrystal covalently linked to DNA [7]. Gold nanoparticles were heated by irradiation of a radiofrequency magnetic field (RFMF), leading to denaturation of a DNA and leaves the surrounding molecules relatively unaffected.

In this study, we propose a novel system for controlling aptamer function by RFMF irradiation (Figure 1). Local heating of a gold nanoparticle by RFMF irradiation would destabilize the aptamer structure and result in a decrease in aptamer function. However, the aptamer can easily refold to its native structure after denaturation of its structure. To evaluate their function easily, we introduced a complementary DNA strand that would not affect aptamer 


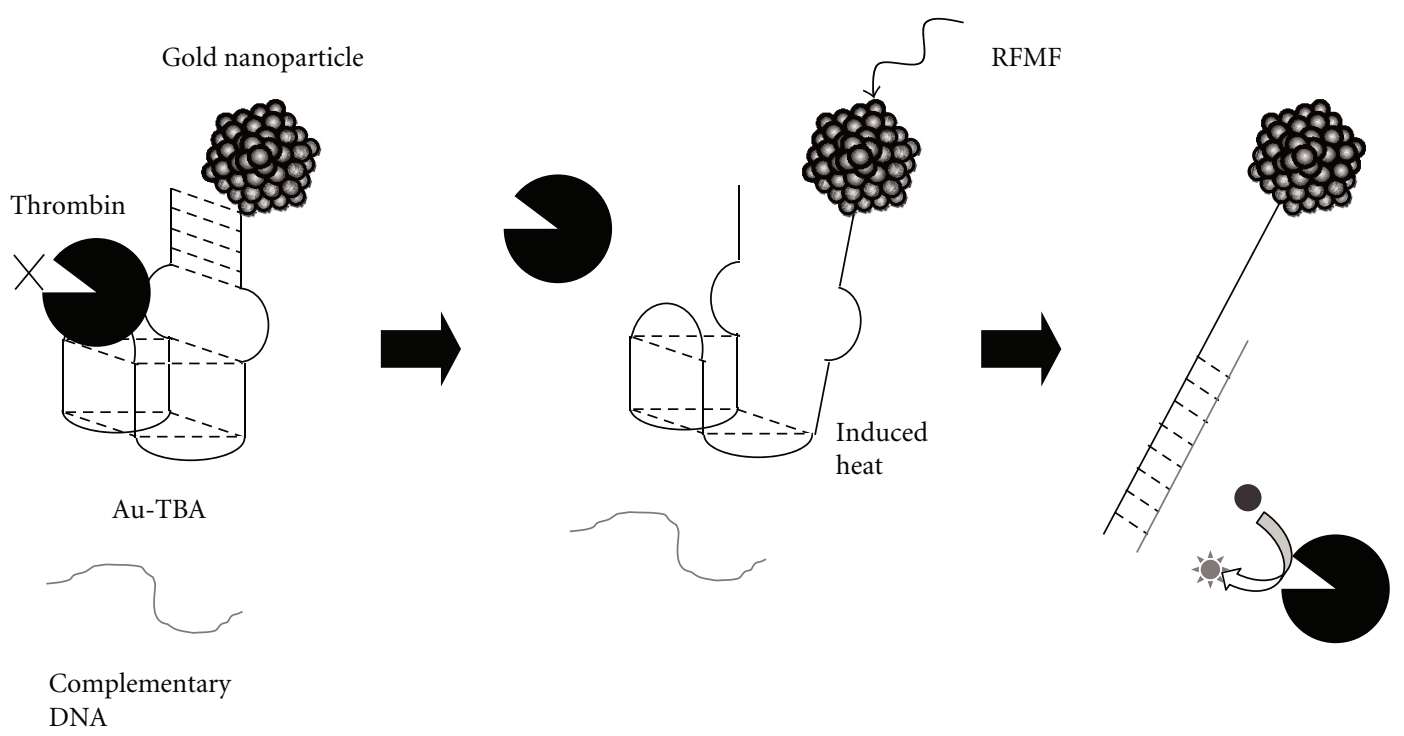

FIGURE 1: The scheme for the controlling of the function of gold nanoparticle-conjugated thrombin-inhibiting aptamer by RFMF.

function in the absence of RFMF irradiation. We expect that the complementary DNA strand hybridizes the aptamer on RFMF irradiation and remains in the hybridized state even after RFMF irradiation. Because the complementary DNA would help repress the refolding of the aptamer to its native structure, we can easily evaluate the aptamer function activity after RFMF irradiation.

In this study, we selected thrombin-binding aptamer (TBA) as a model aptamer. TBA forms a G-quadruplex structure to inhibit thrombin activity [8]. We selected a 31-mer TBA, because it has higher inhibitory activity than the more commonly used 15 -mer TBA $[9,10]$. In addition, because the 31-mer TBA forms the G-quadruplex structure attached to the stem region, we expect that conjugation of the gold nanoparticle has little effect on thrombin inhibitory activity of TBA. We prepared a 31-mer TBA conjugated with a gold nanoparticle (Au-TBA) and examined the control of its function by RFMF irradiation.

\section{Materials and Methods}

2.1. Linkage of Gold Nanocrystals to TBA. A monomaleimide-modified gold nanoparticle (diameter, $1.4 \mathrm{~nm}$ ) was purchased from Nanoprobes (NY, USA). The DNA was synthesized by Operon Biotechnologies (Tokyo, Japan). The $5^{\prime}$ end of the 31-mer TBA ( $5^{\prime}$-CACTGGTAGGTTGGTGTGGTTGGGGCCAGTG-3') was thiolated. A gold nanoparticleconjugated 31-mer TBA was prepared according to the procedure published in the literature [7]. After gold nanoparticles were prepared with $10 \%$ isopropanol and diluted with water, a thiolated 31-mer thrombin-inhibiting aptamer $(3 \mathrm{nmol})$ was coupled with an excess of monomaleimidegold nanoparticles $(30 \mathrm{nmol})$ at $4^{\circ} \mathrm{C}$ for $24 \mathrm{~h}$. A gold nanoparticle-conjugated TBA was purified by Superdex 75
HiLoad 16/60 (GE Healthcare, UK Ltd, Buckinghamshire, England) in $5 \mathrm{mM} \mathrm{NaH}_{2} \mathrm{PO}_{4}, 150 \mathrm{mM} \mathrm{NaCl}$ (pH 6.5).

Successful conjugation of gold nanoparticles with TBA (Au-TBA) was verified by native polyacrylamide gel (11\%) electrophoresis (PAGE) in TBE buffer $(89 \mathrm{mM}$ Tris, $89 \mathrm{mM}$ $\mathrm{H}_{3} \mathrm{BO}_{3}, 2 \mathrm{mM}$ EDTA) at room temperature. The band size of Au-TBA was compared with the fluorescein-modified 31mer TBA. Fluorescein-modified 31-mer TBA was detected by a Typhoon 8600 (GE Healthcare, UK Ltd, Buckinghamshire, England) and Au-TBA was visualized by LI Silver staining (Nanoprobes, NY, USA) for $5 \mathrm{~min}$ to confirm Au-TBA purification.

2.2. Au-TBA Characterization. In TBS buffer $(50 \mathrm{mM}$ Tris$\mathrm{HCl}(\mathrm{pH} 8.0$ ), $100 \mathrm{mM} \mathrm{NaCl}, 5 \mathrm{mM} \mathrm{KCl}$ ), Au-TBA (final concentration, $100 \mathrm{nM}$ ) and 13.5-nM thrombin (Wako Chemicals, Osaka, Japan) were mixed at room temperature for $5 \mathrm{~min}$. Then, $2 \mathrm{mg} / \mathrm{mL}$ fibrinogen (Wako Chemicals, Osaka, Japan) was mixed, and the clotting time was measured using Amelung KC-4A Micro Coagulation Analyzer (RWTUV, Essen, Germany) at $37^{\circ} \mathrm{C}$.

2.3. Evaluation of Complementary DNA to Control Au-TBA Inhibitory Activity. Each complementary DNA (Table 1) $(100 \mathrm{nM})$ was mixed with $\mathrm{Au}-\mathrm{TBA}(100 \mathrm{nM})$ and thrombin $(13.5 \mathrm{nM})$. Mixtures were incubated at room temperature for $5 \mathrm{~min}$ and then mixed with fibrinogen $(2 \mathrm{mg} / \mathrm{mL})$. The clotting time was measured using Amelung KC-4A Micro Coagulation Analyzer at $37^{\circ} \mathrm{C}$.

2.4. Control of the Inhibitory Activity of Au-TBA by RFMF. Au-TBA (100 nM) and 20-mer complementary DNA $(100 \mathrm{nM})$ and thrombin $(13.5 \mathrm{nM})$ were incubated for $30 \mathrm{~s}$ at room temperature in $50 \mathrm{mM}$ Tris- $\mathrm{HCl}(\mathrm{pH} 8.0), 100 \mathrm{mM}$ $\mathrm{NaCl}, 5 \mathrm{mM} \mathrm{KCl}$ and were irradiated by RFMF $(1 \mathrm{GHz}$, 
TABLE 1

\begin{tabular}{llc}
\hline Name & Sequence & Tm value $\left({ }^{\circ} \mathrm{C}\right)^{*}$ \\
\hline TBA & $5^{\prime}$-CACTGGTAGGTTGGTGTGGTTGGGGCCAGTG-3' & 64 \\
14 -mer & $5^{\prime}$-CACTGGCCCCAACC-3' & 52.2 \\
15 -mer & $5^{\prime}$-CACTGGCCCCAACCA-3' & 54.2 \\
$16-$ mer & $5^{\prime}$-CACTGGCCCCAACCAC-3' & 56.4 \\
$17-$ mer & $5^{\prime}$-CACTGGCCCCAACCACA-3' & 58 \\
20 -mer & $5^{\prime}$-CACTGGCCCCAACCACACCA-3' & 63.3 \\
$23-$ mer & $5^{\prime}$-CACTGGCCCCAACCACACCAACC-3' \\
\hline
\end{tabular}

${ }^{*} \mathrm{Tm}$ (melting temperature) values of complementary DNA were predicted using mfold program. Tm value of TBA was measured using UV melting assay.

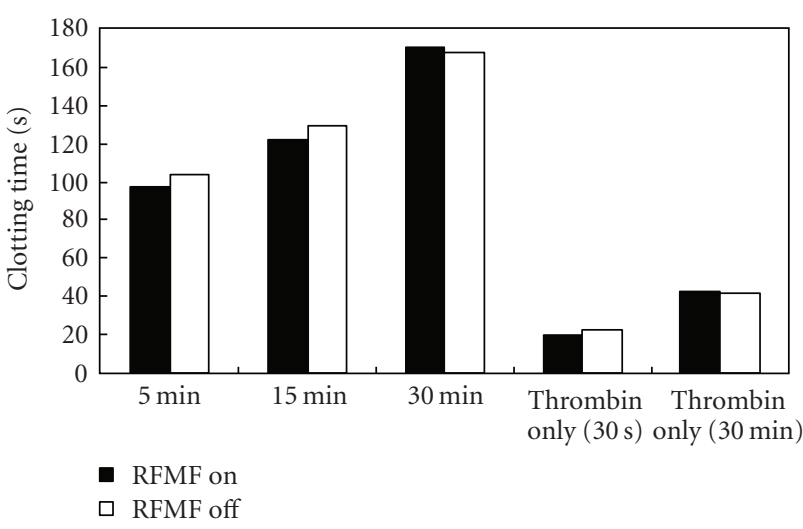

FIGURE 2: Evaluation of the effect of RFMF on thrombin activity. Mixtures of Au-TBA and thrombin were irradiated for $5 \mathrm{~min}$, $15 \mathrm{~min}$, or $30 \mathrm{~min}$. As control, the mixtures were incubated for the same time at room temperature. Fibrinogen was added, and then, the clotting time was measured. White bars show clotting times without RFMF irradiation. Black bars show clotting times with RFMF irradiation.

1.5 W) for 5 min by using an HP 8648C RF signal generator (Hewlett-Packard Company, Calif, USA). The diameter of a coil was $1 \mathrm{~cm}$ and its height was $5 \mathrm{~cm}$ with 35 turns. To optimize RFMF power, we connected a tuning horn to an amplifier for matching. Then, fibrinogen $(2 \mathrm{mg} / \mathrm{mL})$ was mixed, and the clotting time was measured using Amelung KC-4A Micro Coagulation Analyzer at $37^{\circ} \mathrm{C}$.

\subsection{Confirmation of Complementary DNA and $A u-T B A$} Hybridization. Au-TBA (100 nM), 20-mer complementary DNA $(100 \mathrm{nM})$, and thrombin $(13.5 \mathrm{nM})$ were incubated for $30 \mathrm{~s}$ at room temperature in $50 \mathrm{mM}$ Tris- $\mathrm{HCl}(\mathrm{pH} 8.0)$, $100 \mathrm{mM} \mathrm{NaCl}, 5 \mathrm{mM} \mathrm{KCl}$, and some of these samples were irradiated by RFMF $(1 \mathrm{GHz}, 1.5 \mathrm{~W})$ for $5 \mathrm{~min}$ by using the HP 8648C RF signal generator. After the RFMF irradiation, we carried out native polyacrylamide gel electrophoresis (PAGE) using $11 \%$ polyacrylamide gel in TBE buffer $(89 \mathrm{mM}$ Tris, $89 \mathrm{mM} \mathrm{H}_{3} \mathrm{BO}_{3}, 2 \mathrm{mM}$ EDTA) at room temperature. The fluorescence intensities of complementary DNA were analyzed by a Typhoon 8600 .

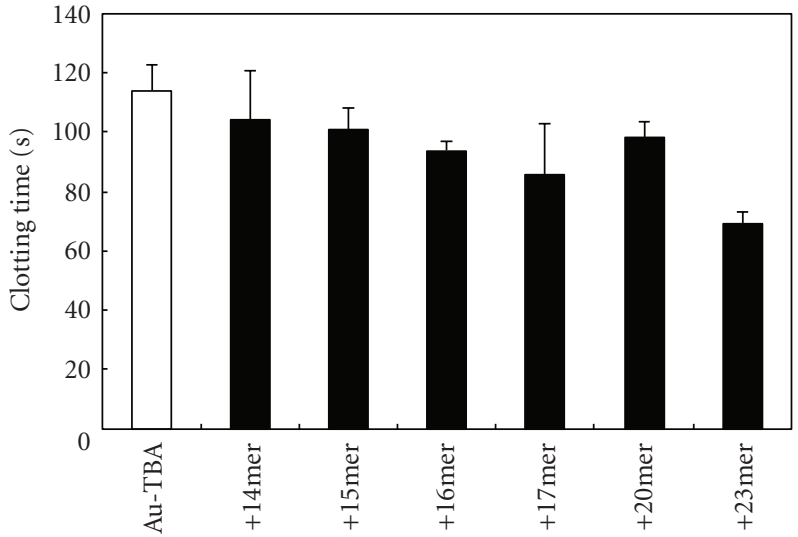

FIGURE 3: Evaluation of the effect of complementary DNA on TBA inhibitory activity. 14-17-mer, 20-mer, and 23-mer complementary DNA $(100 \mathrm{nM})$ were incubated with Au-TBA $(100 \mathrm{nM})$. The white bar shows the clotting time of thrombin and Au-TBA. The gray bar shows the clotting time of thrombin, Au-TBA, and each complementary DNA strand. Each clotting time represents the average of the clotting times in two experiments, and error bars are given.

\section{Results and Discussion}

First, we designed Au-TBA that has RFMF-controllable inhibitory activity. We selected a 31-mer TBA as a model aptamer. The 31-mer TBA forms a G-quadruplex-type structure that is 15-mer TBA attached to 3 base loops and a 5-bp stem. The internal loop region of the Gquadruplex recognizes the thrombin [11]. The stem region would stabilize the G-quadruplex structure and thus increase inhibitory activity. We then selected the $5^{\prime}$ terminal of the aptamer for conjugation with gold nanoparticles and prepared Au-TBA using a monomaleimide gold nanoparticle and $5^{\prime}$ thiol-modified TBA. We expected that the gold nanoparticle would not have an effect on the TBA inhibitory activity; further, heating of the gold nanoparticles by RFMF irradiation destabilizes the stem structure of the aptamer, resulting in a decrease in its inhibitory activity. In fact, the prepared Au-TBA shows inhibitory activity similar to that shown by unmodified TBA. Conjugation of the $5^{\prime}$ terminal of TBA with gold nanoparticles did not significantly affect TBA function (data not shown). 


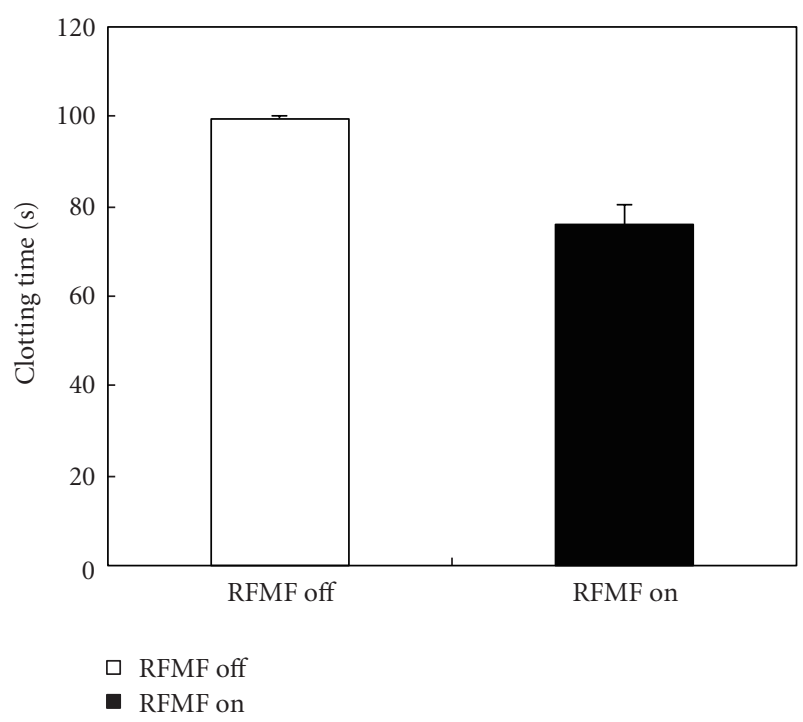

FIgUre 4: The control of the function of Au-aptamer by RFMF. The white bar termed "RFMF off" shows the clotting time of the mixture after 5 min of incubation at room temperature. The black bar termed "RFMF on" shows the clotting time of the mixture of thrombin, fibrinogen, TBA, and 20-mer complementary DNA after 5 min of irradiation using RFMF. Each clotting time represents the average of the clotting times in two experiments, and error bars are given.

Because the heat generated from nanoparticles by RFMF irradiation depends on nanoparticle size and magnetic field frequency, larger gold nanoparticles possibly affect not only the structure of the aptamer, but also thrombin structure. To control aptamer function, we have to select a suitable nanoparticle size and a magnetic field frequency that do not produce an unexpected effect on enzyme activity. Then, we selected gold nanoparticles with a diameter of $1.4 \mathrm{~nm}$, which is the same as that of nanoparticles used in the study of Hamad-Schfferil et al. [7]. They mentioned that the effect of heat induced by this nanoparticle, because $1 \mathrm{GHz}$ RFMF irradiation was limited to proximity. To evaluate the effect of RFMF irradiation on thrombin activity, RFMF was used to irradiate a mixture of Au-TBA and thrombin, and thrombin activity was measured after RFMF irradiation. DNA can easily refold to its correct structure, but it is difficult for proteins to renature correctly. A similar level of inhibition was observed regardless of whether the mixture was irradiated with RFMF (Figure 2). This indicates that heating induced by RFMF irradiation did not affect thrombin activity.

However, it is difficult to evaluate thrombin activity upon RFMF irradiation, because a complicated instrument is required for the measurement of thrombin activity with RFMF irradiation. Then, we used a complementary DNA strand of TBA. We expected RFMF would enhance hybridization between Au-TBA and a complementary DNA strand. The complementary DNA strand would repress refolding of the aptamer to its functional structure after RFMF irradiation, after which one can easily evaluate Au-TBA inhibitory activity. We selected an optimal complementary
DNA strand that insignificantly affects the Au-TBA structure without RFMF irradiation.

First, we investigated the optimal length of the complementary DNA strand for the evaluation of this system. Because the complementary DNA strand should have a Tm value lower than that of TBA $\left(64^{\circ} \mathrm{C}\right)[12]$, we designed $14-$ 17-mer, 20-mer, and 23-mer DNA strands complementary to the $3^{\prime}$ terminal region of TBA; the 14-20-mer was designed such that its $\mathrm{Tm}$ value was lower than that predicted for TBA (Table 1) [13]. When we incubated a 14-17-mer or 20-mer complementary DNA with Au-TBA, they did not significantly affect the inhibitory activity of TBA without RFMF irradiation (Figure 3). On the other hand, the 23-mer complementary DNA decreased the inhibitory activity of TBA. We then selected a 20-mer complementary DNA strand to evaluate the effect of RFMF on the inhibitory activity of TBA.

We evaluated the controlling of TBA inhibitory activity by RFMF irradiation. We mixed thrombin, Au-TBA, and 100-nM 20-mer complementary DNA and incubated these at room temperature for $30 \mathrm{~s}$; this mixture was subsequently subjected to RFMF irradiation for 5 min (Figure 4). Then, we added fibrinogen and measured the clotting time. The thrombin solution containing Au-TBA and 20mer complementary DNA showed a shorter clotting time after RFMF irradiation than after incubation for $5 \mathrm{~min}$ at room temperature without RFMF irradiation (Figure 4). We observed a decrease of about $20 \%$ in the inhibitory activity of TBA by RFMF irradiation.

In order to confirm the successful hybridization of complementary DNA and Au-TBA, we performed native PAGE using fluorescein-modified complementary DNA (FcDNA). Because the gold nanoparticles quench the fluorescence of some fluorescent molecules and the TBA was labeled with a gold nanoparticle, it was difficult to obtain a distinct fluorescence band indicating the formation of complex of Au-TBA and 20-mer complementary DNA. Therefore, we quantified the hybridization reaction by comparing the fluorescence intensity of free FcDNA. We observed a decrease in the fluorescence intensity when Au-TBA was mixed with FcDNA (Figure 5(a), lane 2) rather than when FcDNA was used alone (Figure 5(a), lane 1) after RFMF irradiation. Although this decrease was also observed without RFMF (Figure 5(a), lane 3), we observed a weaker fluorescence intensity for free FcDNA subjected to RFMF irradiation than FcDNA that was not subjected to the same. The ratio of decrease in fluorescence intensity was approximately $20 \%$ (Figure 5(b)), and it shows good agreement with inhibitory activity results. These results indicate that RFMF irradiation destabilizes the Au-TBA structure and results in acceleration of hybridization between a complementary DNA strand and Au-TBA. We can say that we were able to control the inhibitory activity of TBA with complementary DNA and RFMF irradiation.

RFMF irradiation might induce heating of Au nanoparticle resulting in destabilization of Au-TBA structure. During RFMF irradiation, hybridization of complementary DNA with Au-TBA might compete with refolding to native structure of Au-TBA. Since RFMF irradiation would be limited 


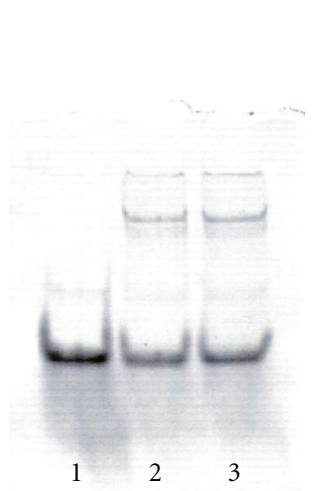

(a)

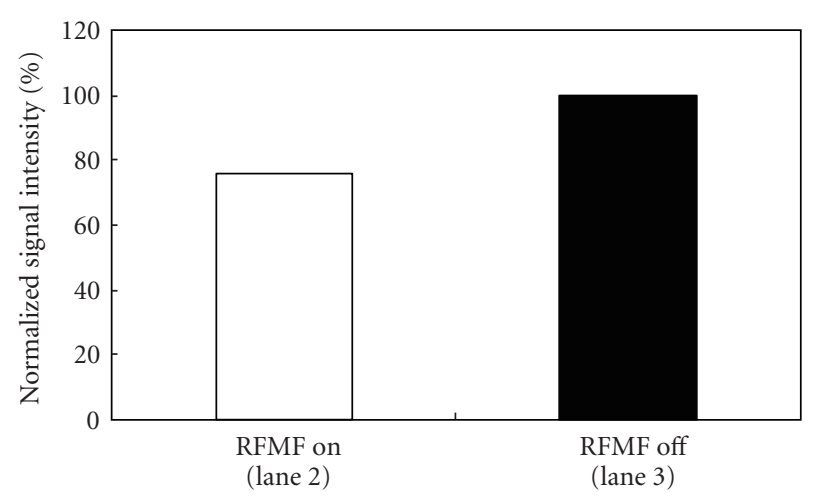

(b)

FIGURE 5: The confirmation of hybridization between FCDNA and Au-TBA by native PAGE. (a) Lane 1 shows a fluorescence image of 20mer FcDNA. Lane 2 shows a fluorescence image of FcDNA with thrombin and Au-TBA after RFMF irradiation. Lane 3 shows a fluorescence image of FcDNA with thrombin and Au-TBA after incubation for $5 \mathrm{~min}$ at room temperature. (b) Signal intensity of FcDNA of Lane 2 and 3 normalized to Lane 2.

to local area around Au nanoparticle, induced heating of Au nanoparticle would not have so much effect on the solution temperature. Therefore, free Au-TBA might refold to native structure, whereas duplex might keep their structures after RFMF irradiation. Additionally, complementary DNA strand concentration would affect hybridization efficiency. Therefore, the control of structure of Au-TBA would be improved by adjusting of RFMF irradiation time and complementary DNA strand concentration.

In order to apply this system in therapeutic medicine, we should be able to control aptamer function by RFMF without the need for a complementary DNA strand. Because RFMF irradiation would destabilize the aptamer structure, the function of the aptamer is possibly repressed by RFMF irradiation in the absence of a complementary DNA strand. It may not be easy to determine the exact effect of RFMF irradiation on the aptamer function without a complementary DNA strand, but we can control it by adjusting the RFMF frequency and size of the gold nanoparticle, because the extent of instability of the structure of the gold nanoparticleconjugated aptamer would depend on the energy generated from the gold nanoparticle after RFMF irradiation.

Control of aptamer function by RFMF irradiation has the following 3 advantages over that by UV light irradiation: in vivo penetration, no DNA damage, and limited effect on conjugated DNA. Although there are many reports describing the remote control of aptamers by UV light, deep tissue penetration is difficult and limits their application in vitro or requires two-photon absorption, which can be achieved only by a specific device. On the other hand, because RFMF can penetrate deep tissues, its application is not limited to an in vitro setting and can also be used in vivo. In addition, because the effect of heating induced by gold nanoparticles irradiated by RFMF is limited to the structure of the aptamer conjugated with gold nanoparticles, the heating of gold nanoparticles breaks the tertiary structure of the aptamer but does not modify its chemical structure. In addition, the heating of an Au nanoparticle can be limited to a local region by adjusting the frequency of RFMF and size of the gold nanoparticle [7]. Therefore, RFMF irradiation prevents the unexpected effect caused by DNA damage and excessive heating of gold nanoparticles.

\section{Conclusions}

When therapeutic medicine produces an unexpected effect, it becomes necessary to control therapeutic medicine. Although antidotes based on complementary DNA have been developed for the use of therapeutic aptamers [14], remote control in therapeutic medicine is an attractive strategy. RFMF is widely used for magnetic resonance imaging and photothermal therapy $[15,16]$. Therefore, heating induced by gold nanoparticles irradiated by RFMF is an attractive option for controlling aptamer function remotely in vivo. In this study, we demonstrated the control of thrombin inhibitory activity of TBA by RFMF irradiation and the use of using complementary DNA. Although we should develop a control system that does not require complementary DNA, we expect that the controlling of aptamer function by RFMF would be a useful tool in the future.

\section{Authors' Contributions}

Both K. Taira and K. Abe equally contributed to this paper.

\section{Acknowledgment}

This work is partly supported by the Grant-in-Aid for the 21st Century COE "Future Nano-Materials" from the Ministry of Education, Culture, Sports, Science, and Technology (MEXT) of Japan.

\section{References}

[1] A. D. Ellington and J. W. Szostak, "In vitro selection of RNA molecules that bind specific ligands," Nature, vol. 346, no. 6287, pp. 818-822, 1990. 
[2] C. Tuerk and L. Gold, "Systemic evolution of ligands by exponential enrichment: RNA ligands to bacteriophage T4 DNA polymerase," Science, vol. 249, no. 4968, pp. 505-510, 1990.

[3] T. Hermann and D. J. Patel, "Adaptive recognition by nucleic acid aptamers," Science, vol. 287, no. 5454, pp. 820-825, 2000.

[4] V. Mikat and A. Heckel, "Light-dependent RNA interference with nucleobase-caged siRNAs," RNA, vol. 13, no. 12, pp. 2341-2347, 2007.

[5] M. C.R. Buff, F. Schäfer, B. Wulffen et al., "Dependence of aptamer activity on opposed terminal extensions: improvement of light-regulation efficiency," Nucleic Acids Research, vol. 38, no. 6, pp. 2111-2118, 2009.

[6] K. Konig, "Multiphoton microscopy in life sciences," Journal of Microscopy, vol. 200, no. 2, pp. 83-104, 2000.

[7] K. Hamad-Schifferli, J. J. Schwartz, A. T. Santos, S. Zhang, and J. M. Jacobson, "Remote electronic control of DNA hybridization through inductive coupling to an attached metal nanocrystal antenna," Nature, vol. 415, no. 6868, pp. 152-155, 2002.

[8] L. C. Bock, L. C. Griffin, J. A. Latham, E. H. Vermaas, and J. J. Toole, "Selection of single-stranded DNA molecules that bind and inhibit human thrombin," Nature, vol. 355, no. 6360, pp. 564-566, 1992.

[9] K. Ikebukuro, Y. Okumura, K. Sumikura, and I. Karube, "A novel method of screening thrombin-inhibiting DNA aptamers using an evolution-mimicking algorithm," Nucleic Acids Research, vol. 33, no. 12, p. e108, 2005.

[10] A. B. Dobrovolsky, E. V. Titaeva, S. G. Khaspekova, V. A. Spiridonova, A. M. Kopylov, and A. V. Mazurov, "Inhibition of thrombin activity with DNA-aptamers," Bulletin of Experimental Biology and Medicine, vol. 148, no. 1, pp. 33-36, 2009.

[11] K. Padmanabhan, K. P. Padmanabhan, J. D. Ferrara, J. E. Sadler, and A. Tulinsky, "The structure of $\alpha$-thrombin inhibited by a 15-mer single-stranded DNA aptamer," Journal of Biological Chemistry, vol. 268, no. 24, pp. 17651-17654, 1993.

[12] K. Abe, D. Ogasawara, W. Yoshida, K. Sode, and K. Ikebukuro, "Aptameric sensors based on structural change for diagnosis," Faraday Discussions, vol. 149, pp. 93-105, 2011.

[13] N. R. Markham and M. Zuker, "DINAMelt web server for nucleic acid melting prediction," Nucleic Acids Research, vol. 33, no. 2, pp. W577-W581, 2005.

[14] C. P. Rusconi, J. D. Roberts, G. A. Pitoc et al., "Antidotemediated control of an anticoagulant aptamer in vivo," Nature Biotechnology, vol. 22, no. 11, pp. 1423-1428, 2004.

[15] A. Ito, Y. Kuga, H. Honda et al., "Magnetite nanoparticleloaded anti-HER2 immunoliposomes for combination of antibody therapy with hyperthermia," Cancer Letters, vol. 212, no. 2, pp. 167-175, 2004.

[16] J. Cardinal, J. R. Klune, E. Chory et al., "Noninvasive radiofrequency ablation of cancer targeted by gold nanoparticles," Surgery, vol. 144, no. 2, pp. 125-132, 2008. 

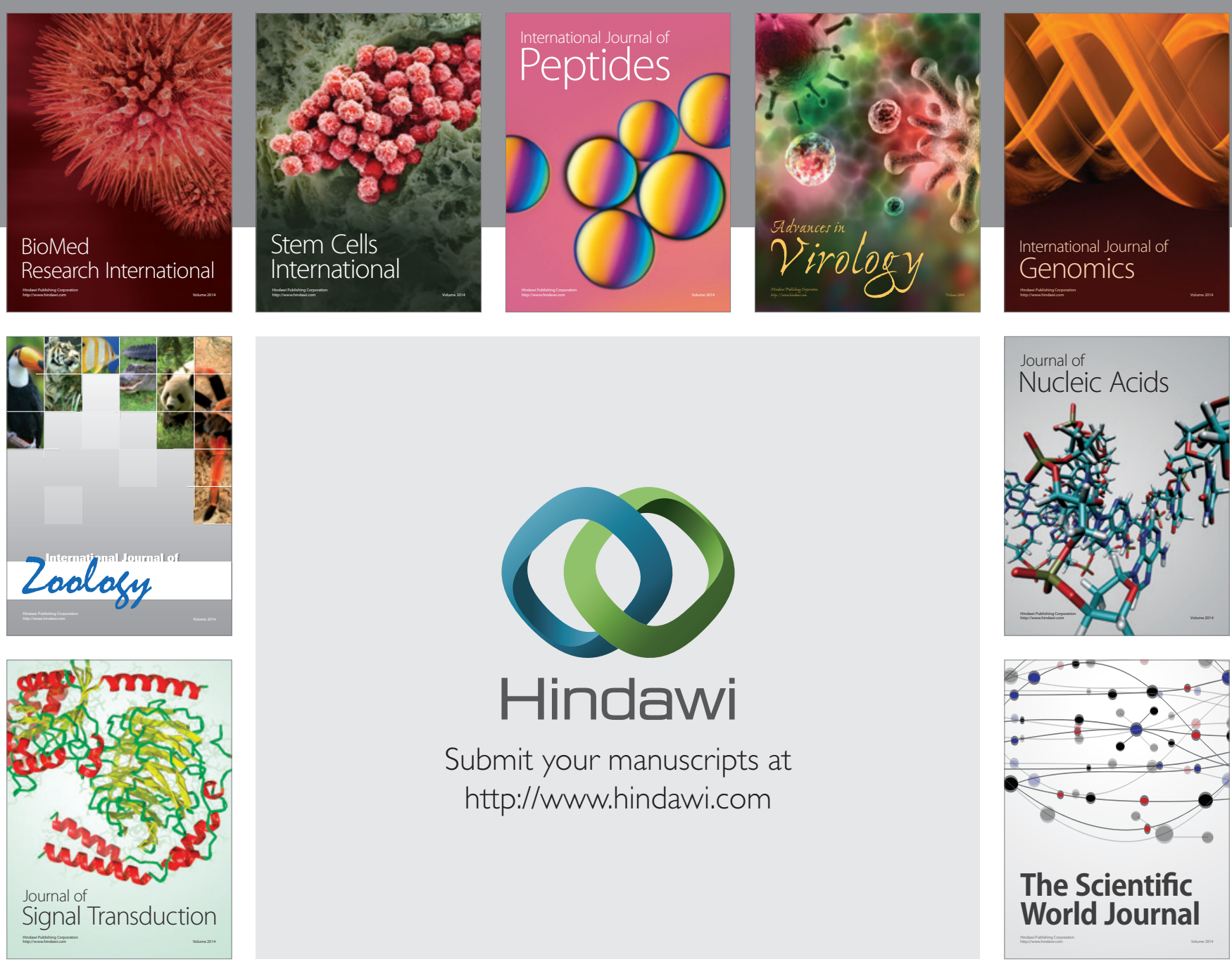

Submit your manuscripts at

http://www.hindawi.com
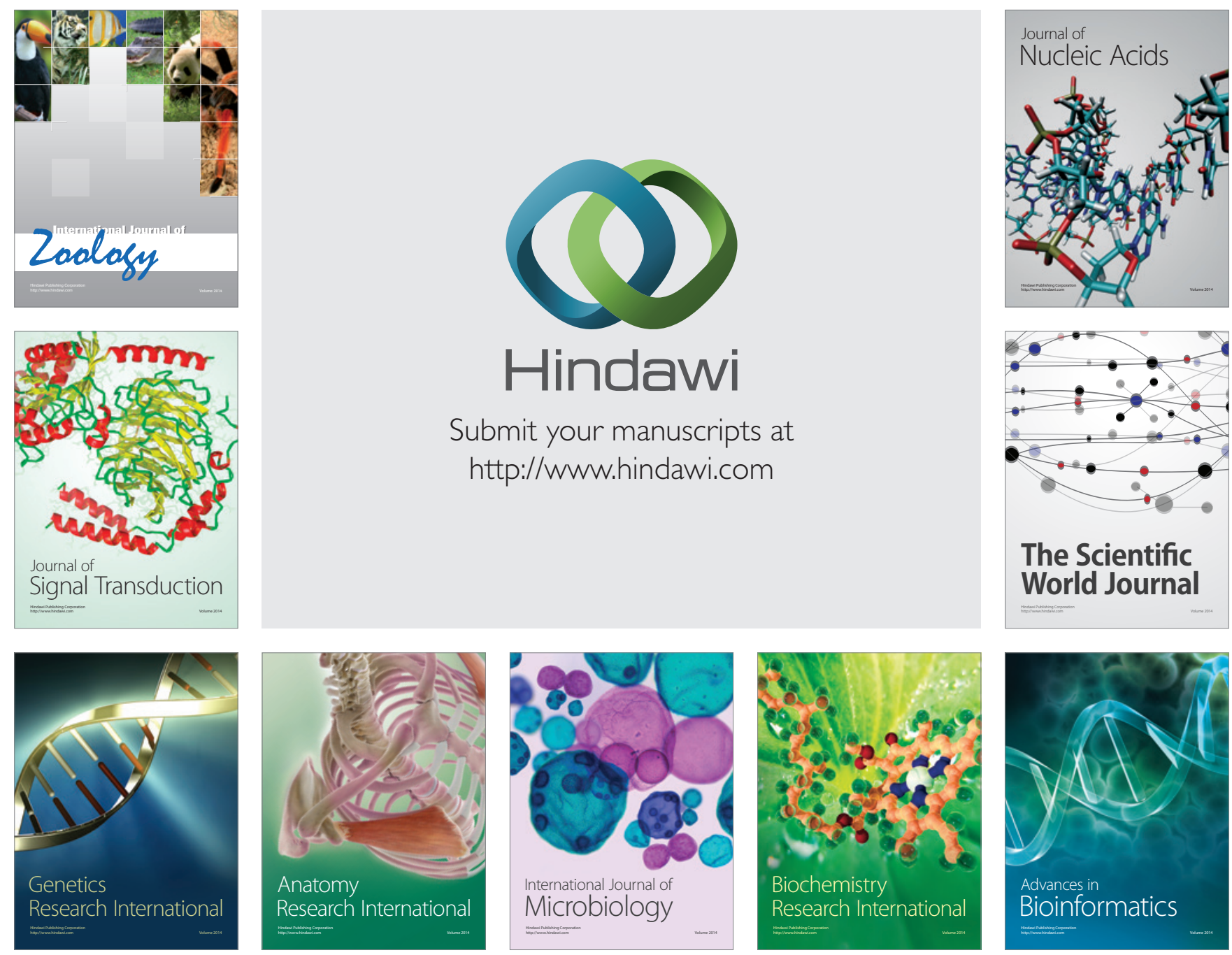

The Scientific World Journal
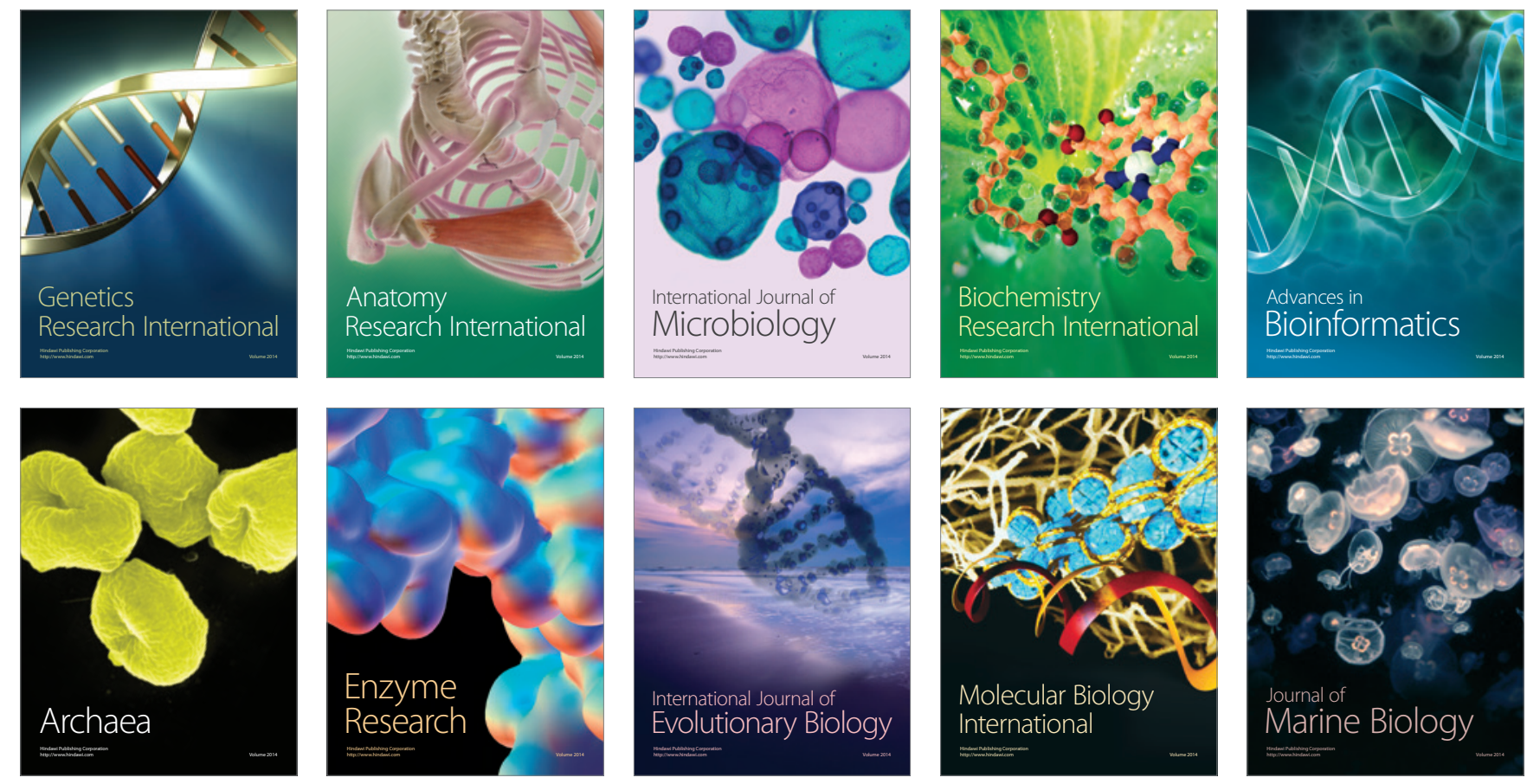